\title{
Roughness scattering in X-ray grazing-incidence telescopes
}

\author{
F. E. Zocchi
}

\author{
Media Lario Technologies, Località Pascolo, 23842 Bosisio Parini (LC), Italy \\ e-mail: fabio.zocchi@media-lario.com
}

Received 11 December 2008 / Accepted 26 February 2009

\section{ABSTRACT}

\begin{abstract}
In the framework of the scalar theory of diffraction, we determine the rms width of the angle spread function degraded by surface roughness in reflective grazing-incidence optics of cylindrical symmetry for X-ray astronomy. Our derivation does not rely on the small roughness approximation, is thus valid for arbitrary roughness values, and takes into full consideration the cylindrical symmetry of the mirrors. The dependence of the rms beam width on both the grazing-incidence angles and the radiation wavelength is studied for both single and double reflection optics, the latter being more relevant to X-ray telescopes. When the small roughness approximation is applicable, we also derive an expression for the angle spread function of the optical system in the presence of roughness scattering that provides the correct expression for the rms beam width.
\end{abstract}

Key words. X-rays: general - telescopes - scattering

\section{Introduction}

A factor limiting the resolution of grazing-incidence optics for $\mathrm{X}$-ray astronomy is the scattering caused by the surface roughness of the telescope mirrors. A number of X-ray space missions (Aschenbach 1988; Citterio et al. 1988; Gondoin et al. 1994; Weisskopf et al. 2000; Gehrels et al. 2004; Pareschi \& Ferrando 2005; Bookbinder et al. 2008; Friedrich et al. 2008) have stimulated both theoretical and experimental research (Spiller 1994; Church \& Takacs 1995, 1993; Harvey 1995a; Harvey et al. 1996; Stearns et al. 1998; Harvey 1995b; Christensen et al. 1988; Karabacak et al. 2000; Zhao \& Spreybroeck 2003) to understand and characterize in a more robust way the scattering of electromagnetic waves from rough surfaces. The typical resolution requirement at the focal plane of an X-ray telescope is sharper than $20^{\prime \prime}$ half energy width at X-ray photon energy between $0.1 \mathrm{keV}$ and $80 \mathrm{keV}$, depending on the mission (Gondoin et al. 1994; Pareschi \& Ferrando 2005; Friedrich et al. 2008), and at a grazing-incidence angle in the range between about $0.1^{\circ}$ and $1.5^{\circ}$. Consequently, the contribution of roughness to the overall optical resolution is sufficiently low to require reflective surfaces polished down to roughness of about $0.2 \div 0.5 \mathrm{~nm} \mathrm{rms}$, typically in the spatial frequency range between $0.01 \mu \mathrm{m}^{-1}$ and $1 \mu \mathrm{m}^{-1}$. Hence, the correlation between surface roughness and radiation scattering is essential to defining the surface quality required by the mirrors.

The interest in the field has been further boosted by other applications of the short-wavelength region of the electromagnetic spectrum, including EUV microlithography (Schellenberg 2008; Bakshi 2008) and EUV microscopy (Silk 1980; Foltyn et al. 2004; Schäfer et al. 2006), just to quote a few. The development of multilayer structures (Barbee 1986; Joensen et al. 1995; Yamashita et al. 1999; Spiller 2003) to enhance the optical reflectivity of mirrors at short wavelengths has also stimulated theoretical and experimental investigations in the field.

The configuration of choice for the optical design of X-ray telescopes is based on the type I Wolter architecture (Wolter $1952 b$ ) in which the X-ray radiation from distant sources is first reflected by a parabolic surface and then by a hyperboloid, both with cylindrical symmetry around the optical axis. The two surfaces are arranged in a coaxial configuration and share a common focus. The second focus of the hyperboloid is the image focus. To maximize the energy throughput, the telescope consists of a multitude of these double-reflection mirrors arranged in a nested configuration. All mirrors operate at grazing-incidence angles low enough to assure high reflectivity from the coating material at X-ray wavelengths. A mirror based on the Wolter architecture is known to satisfy approximately Abbe's condition (Abbe 1873; Braat 1997), assuring limited coma aberration for moderate field of view. A variation of the Wolter telescope, the Wolter-Schwarzschild configuration (Wolter 1952a; Chase \& Speybroeck 1973), exactly satisfies Abbe's condition. Other designs are possible (Giacconi et al. 1969; Zocchi \& Vernani 2007) but they all share the double-reflection feature at very small grazing-incidence angle.

Although a significant number of scientific publications have appeared in which the degradation of the performance of optical systems by scattering from surface roughness has been analyzed, the theoretical determination of the angular width of the scattered beam has received much less attention. The relation between the half-energy width in grazing-incidence telescopes and the roughness variance was studied in (Spiga 2007) by applying the Debye-Waller expression for the total integrated scattering (Spiller 1994) in 1-dimensional geometry. In the framework of the scalar theory of diffraction (Harvey 1995b; Goodman 1968; Gaskill 1978; Born \& Wolf 1980), an exact derivation of the rms width of the angle spread function from the corresponding optical transfer function (Goodman 1968; Gaskill 1978; Born \& Wolf 1980; Smith 1963; Harvey et al. 1988) in 2-dimensional geometry was reported in (Zocchi 2009), in which the contribution of scattering to the rms width of the angle spread function was shown to depend on only the variance of the roughness slope. However, even in these two papers (Spiga 2007; Zocchi 2009), the cylindrical symmetry of an X-ray telescope was not taken into account.

In this work, the rms width of the angle spread function of grazing-incidence optics with cylindrical symmetry is derived for arbitrary values of the roughness variance in the framework 
of the scalar theory of Fourier diffraction. In the following, we simply refer to the rms width of the angle spread function as the beam width. No further approximations are introduced into the derivation apart from the assumption that the angle between the axial profile of the optics and the optical axis is small. It is found again that the contribution of scattering to the width of the angle spread function depends only on the variance of the roughness slope. However, in the extreme case in which both the tapering angle of the optics and the grazing-incidence angle are small, the proportionality factor between the roughness slope and the beam width is half of that found for the reflection on an almost flat surface.

When the small roughness approximation is valid, it is also shown that the angle spread function can be written in a standard form as the convolution of the angle spread function of a perfectly smooth optics with an effective power spectral density expressed by a line integral of the true power spectral density of surface roughness of the mirrors. Finally, for an isotropic powerlaw dependence of the power spectral density on the spatial frequency, the behavior of the beam width as a function of the radiation wavelength $\lambda$ and the grazing-incidence angle $\theta$ is also studied. If $m$ is the exponent of the power-law power spectral density, the contribution of scattering to the beam width is proportional to $\lambda^{(m-6) / 2} \sin ^{2-m / 2} \theta$.

The paper is organized as follows. Section 2 briefly reviews the standard approach used to derive the angle spread function of a rough mirror in the framework of Fourier optics within the small angle approximation, when the angle between the principal ray and any other rays in the optical system is small. This approximation limits the application to mirrors of low ratio of the diameter to the radius of curvature. The results and methods summarized in Sect. 2 are then used in Sect. 3 to derive the width of the angle spread function in grazing-incidence optics with cylindrical symmetry. Finally, in Sect. 4 we derive an expression for the angle spread function when the small roughness approximation is valid. In this last section, the dependence of the beam width on the radiation wavelength and the grazingincidence angle is also studied for an isotropic surface characterized by a power-law expression for the power spectral density. The paper ends with two Appendixes. The first presents some mathematical derivations, and in the second we discuss the range of validity of Fourier optics treatment of scattering from surface roughness, and consequently of the content of this paper, and its relation to the small roughness approximation.

\section{Angle spread function of rough optics}

In this section, we briefly review the treatment of radiation scattering from surface roughness in the framework of the scalar theory of diffraction (Goodman 1968; Gaskill 1978; Born \& Wolf 1980; Smith 1963; Harvey et al. 1988). In this theory, the optical transfer function $H(f)$ of the imaging system is given by the normalized autocorrelation of the generalized complex pupil function $A(\boldsymbol{r})$ in the exit pupil plane (Stearns et al. 1998; Harvey 1995b),

$H(\boldsymbol{f})=\hat{H}(\lambda \boldsymbol{f})=\int A(\boldsymbol{r}) A^{*}(\boldsymbol{r}+\lambda \boldsymbol{f}) \mathrm{d} \boldsymbol{r}$,

where $\lambda$ is the wavelength of the electromagnetic radiation and where $|A(\boldsymbol{r})|^{2}$ is assumed to be normalized to unit integral. Equation (1) also defines a function $\hat{H}(\boldsymbol{r})$ for later use. The description of the behavior of an optical system by Eq. (1) is valid if the pupil function can be considered independent of the position of the object point (Born \& Wolf 1980). In the simplest case, $A(\boldsymbol{r})$ is just the pupil function multiplied by the surface reflectance or transmittance but it may also include a phase factor describing the optical aberrations of the system. The effect of surface roughness can also be included in $A(\boldsymbol{r})$ as a phase factor. When the radiation is incident on the reflecting surface at near normal incidence, the complex pupil function can be written as

$A(\boldsymbol{r})=A_{0}(\boldsymbol{r}) \mathrm{e}^{\frac{4 \pi \mathrm{i}}{\lambda} h(\boldsymbol{r})}$

where $h(\boldsymbol{r})$ is the height of the surface roughness at position $\boldsymbol{r}$ and $A_{0}(\boldsymbol{r})$ is the complex pupil function of the optical system when roughness is neglected. When off-normal incidence is considered with a grazing-incidence angle $\theta$, Eq. (2) must be modified accordingly. Assuming the exit pupil plane to be normal to the principal ray, $\theta$ is also the angle between the normal to the pupil plane and the normal to the rough surface (Stearns et al. 1998). The height of surface roughness is projected onto the normal to the pupil plane by means of a factor $\sin \theta$. In addition, distances across the optical surface are also projected onto the pupil plane by a factor $\sin \theta$ (Stearns et al. 1998; Harvey 1995b). Hence, Eq. (2) becomes

$A(\boldsymbol{r})=A_{0}(\boldsymbol{r}) \mathrm{e}^{\frac{4 \pi \mathrm{i} \sin \theta}{\lambda} h\left(\frac{r_{1}}{\sin \theta}, r_{2}\right)}$,

where $r_{1}$ and $r_{2}$ are the components of $\boldsymbol{r}$ and where, for definiteness, the plane of incidence is assumed along the 1-axis. The effect of roughness is obtained by substituting Eq. (3) into Eq. (1) and by averaging the result over the roughness height (Stearns et al. 1998; Harvey 1995b), to obtain

$$
\begin{aligned}
H(\boldsymbol{f})= & \int A_{0}(\boldsymbol{r}) A_{0}^{*}(\boldsymbol{r}+\lambda \boldsymbol{f}) \\
& \times\left\langle\mathrm{e}^{\frac{4 \pi \mathrm{i} \sin \theta}{\lambda}\left[h\left(\frac{r_{1}}{\sin \theta}, r_{2}\right)-h\left(\frac{r_{1}+\lambda f_{1}}{\sin \theta}, r_{2}+\lambda f_{2}\right)\right]}\right\rangle \mathrm{d} \boldsymbol{r} .
\end{aligned}
$$

The final step consists in assuming that $h(\boldsymbol{r})$ is a Gaussian stationary and ergodic stochastic process (Papoulis 1965) and in ignoring multiple scattering and shadowing effects (Stearns et al. 1998; Harvey 1995b). Without loss of generality, we can remove the average of $h(\boldsymbol{r})$ and include it into the deterministic phase factor of $A_{0}(\boldsymbol{r})$. A standard calculation of multivariate Gaussian casual variables then gives (Papoulis 1965)

$$
\left\langle\mathrm{e}^{\frac{4 \pi \mathrm{i} \sin \theta}{\lambda}\left[h\left(\frac{r_{1}}{\sin \theta}, r_{2}\right)-h\left(\frac{r_{1}+\lambda f_{1}}{\sin \theta}, r_{2}+\lambda f_{2}\right)\right]}\right\rangle=\mathrm{e}^{-\frac{16 \pi^{2} \sin ^{2} \theta}{\lambda^{2}}\left[\sigma^{2}-C\left(\frac{\lambda f_{1}}{\sin \theta}, \lambda f_{2}\right)\right]}
$$

where $C(\boldsymbol{r})$ is the covariance of surface roughness and $\sigma^{2}=C(0)$ is the corresponding variance. Because of the assumption that the stochastic process is stationary and ergodic, the right-hand side of Eq. (5) is independent of $\boldsymbol{r}$. In addition, in the small angle approximation, when the angle between the principal ray and any other rays in the optical system is small, the angle $\theta$ can also be considered to be independent of $\boldsymbol{r}$ (Stearns et al. 1998; Harvey 1995b). Hence, Eq. (5) can be taken out of the integral in Eq. (4) and the optical transfer function simplifies to

$H(\boldsymbol{f})=H_{0}(\boldsymbol{f}) \mathrm{e}^{-\frac{16 \pi^{2} \sin ^{2} \theta}{\lambda^{2}}\left[\sigma^{2}-C\left(\frac{\lambda f_{1}}{\sin \theta}, \lambda f_{2}\right)\right],}$

where $H_{0}(f)$ is the optical transfer function of the system when roughness is or can be ignored. Before proceeding, we summarize the conditions under which Eq. (6) holds: a) the angle between any ray in the optical system and the principal ray is small; b) multiple scattering and shadowing effects can be ignored; c) the pupil function is independent of the position of the 

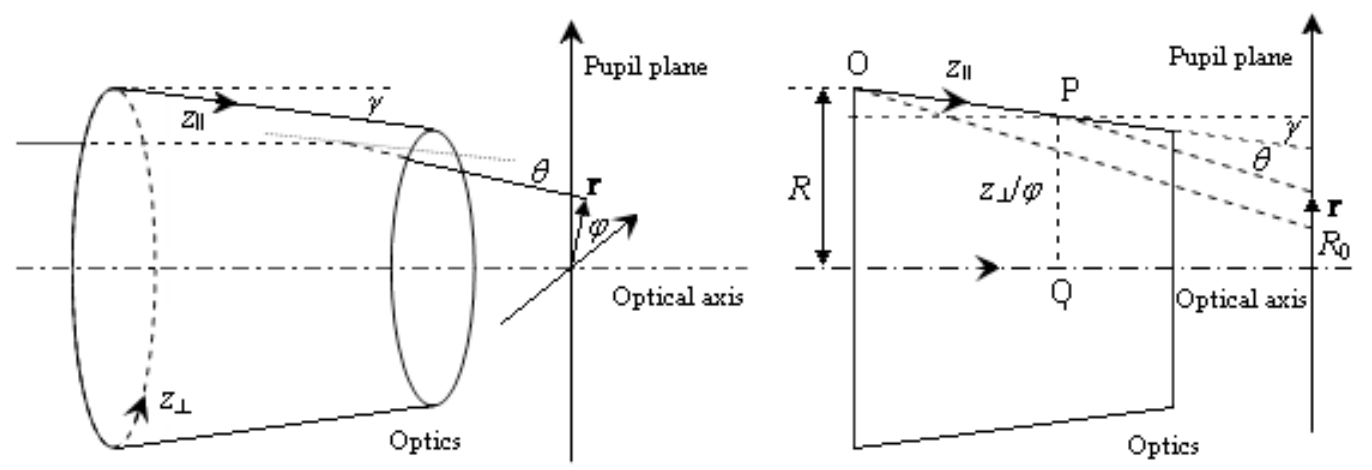

Fig. 1. Geometry of the grazing-incidence optics with cylindrical symmetry: conceptual 3D drawing (left) anf optics cross section (right).

object point; d) surface roughness can be described by a stationary ergodic 2-dimensional stochastic process.

The inverse Fourier transform of the optical transfer function is the angle spread function (Harvey 1995b). When dealing with scattering phenomena, the use of the angle spread function is more convenient than the point spread function since the former is also applicable to large scattering angles (Harvey 1995a,b). In this formalism, the Fourier variable conjugate to the dimensionless frequency vector $\boldsymbol{f}$ appearing in Eq. (6) is the vector of direction cosines $\alpha$ of components $\alpha_{1}$ and $\alpha_{2}$ in the image plane. Because of the Fourier transform relation between the optical transfer function $H(f)$ and the angle spread function $P(\alpha)$, the integral of the latter is normalized to unity,

$$
\int P(\alpha) \mathrm{d} \alpha=H(0)=1
$$

The integration in the above equation should be performed in the domain $\alpha=|\alpha| \leq 1$ but, in this case, the integral would no longer be equal to $H(0)$. We, instead, assume that $P(\alpha)$ decreases sufficiently rapidly with $\alpha$ for the integration to be extended over the entire plane of the direction cosines. The radiant intensity per unit solid angle $I(\alpha)$ is related to $P(\alpha)$ by $I(\alpha)=P(\alpha) \sqrt{1-\alpha^{2}}$ (Harvey 1995b). Thus, the total radiant power is also normalized to unity,

$$
\int I(\alpha) \mathrm{d} \Omega=\int P(\alpha) \mathrm{d} \alpha=1,
$$

where the first equality follows by taking into account the expression $\mathrm{d} \Omega=\mathrm{d} \alpha / \sqrt{1-\alpha^{2}}$ for the infinitesimal solid angle $\mathrm{d} \Omega$.

The width of the reflected and scattered beam can be defined to be the root mean square value of the scattering angle weighted by the angle spread function,

$\delta=\left(\int \alpha^{2} P(\boldsymbol{\alpha}) \mathrm{d} \boldsymbol{\alpha}\right)^{1 / 2}$.

The full angular width of the beam is then $2 \arcsin \delta$. In the following, we simply refer to $\delta$ as the angular width of the beam. It is again assumed that the angle spread function $P(\alpha)$ decreases fast enough at high value of $|\alpha|$ to extend the integration in Eq. (7) over the whole plane of the direction cosines. Because of the Fourier transform relation between the optical transfer function and the angle spread function, the integral in Eq. (7) can be expressed in terms of the value at the origin of the derivatives of $H(\boldsymbol{f})$. Since the inverse Fourier transform of $\Delta H(\boldsymbol{f})$ is $-4 \pi^{2} \alpha^{2} P(\alpha)$, the integral in Eq. (7) is

$\int \alpha^{2} P(\boldsymbol{\alpha}) \mathrm{d} \boldsymbol{\alpha}=-\left.\frac{1}{4 \pi^{2}} \Delta H(\boldsymbol{f})\right|_{f=0}$, where $\Delta$ is the 2-dimensional Laplace operator. Inserting Eq. (8) into Eq. (7), we obtain

$\delta=\frac{1}{2 \pi} \sqrt{-\left.\Delta H(f)\right|_{f=0}}$.

The right-hand side of Eq. (9) can be calculated in terms of $H_{0}(f)$ and the power spectral density $S(\boldsymbol{v})$ of surface roughness by means of Eq. (6). The result, as reported in Zocchi (2009), is

$\delta^{2}=\delta_{0}^{2}+4 \rho_{\|}^{2}+4 \rho_{\perp}^{2} \sin ^{2} \theta$,

where

$\begin{aligned} \rho_{\|}^{2} & =4 \pi^{2} \int S(v) v_{\|}^{2} \mathrm{~d} v, \\ \rho_{\perp}^{2} & =4 \pi^{2} \int S(v) v_{\perp}^{2} \mathrm{~d} v,\end{aligned}$

are the variances of roughness slope along the direction of the incidence plane and normal to the incidence plane, respectively. In Eq. (10), $\delta_{0}=\left(-\left.\Delta H_{0}(\boldsymbol{f})\right|_{f=0}\right)^{1 / 2} / 2 \pi$ is the rms beam width when roughness is ignored. Thus, $\delta_{0}$ only takes into account the effects of diffraction and aberrations in the optical surface. The power spectral density $S(v)$ in Eqs. (11) and (12) is defined as the bilateral Fourier transform of the covariance $C(\boldsymbol{r})$.

\section{Width of the angle spread function in X-ray telescopes}

The results presented in the previous section are valid for almost flat mirrors. The theory is now extended to the case of grazingincidence optics with cylindrical symmetry. We still assume the validity of the small angle approximation: in each plane containing the optical axis, the angle between any two rays reflected along a mirror profile is much smaller than the average grazingincidence angle.

The analysis is based on the geometry shown in Fig. 1. It is assumed that the grazing-incidence optics consists of a mirror with just one reflection that can be approximated by a cone forming an angle $\gamma$ with the optical axis. The extension to two or more reflections will be considered later. The rays reflected from the optics form a grazing-incidence angle $\theta$ with the optical surface and are contained in planes through the optical axis. The exit pupil plane is perpendicular to the optical axis, which is also the axis of the cone. The largest radius $R$ of the cone is projected onto the exit pupil plane as a circle of radius $R_{0}$. The position on the optical surface is defined by a coordinate $z_{\|}$along a profile 
of the cone and a coordinate $z_{\perp}$ along circles at the intersection of the optical surface with planes normal to the optical axis. In addition, we form the vector $z$ of components $z_{\|}$and $z_{\perp}$. We use polar coordinates in the pupil plane: with a point in this plane identified by a position vector $\boldsymbol{r}$, we associate its modulus $r$ and its anomaly $\varphi$. We finally assume that the pupil function $A_{0}(\boldsymbol{r})$ has cylindrical symmetry and depends only on the modulus $r$ of $r$.

The relation $z=\boldsymbol{z}(\boldsymbol{r})$ between the vector $\boldsymbol{z}$ on the optical surface and the vector $\boldsymbol{r}$ on the pupil plane is obtained by inspection of Fig. 1 as

$z=z(\boldsymbol{r})=\left\{\begin{array}{l}z_{\|}=\mathrm{P}-\mathrm{O}=\frac{\cos (\theta+\gamma)}{\sin \theta}\left(|\boldsymbol{r}|-R_{0}\right) \\ z_{\perp}=(\mathrm{P}-\mathrm{Q}) \angle \boldsymbol{r}=\left(R+z_{\|} \sin \gamma\right) \angle \boldsymbol{r}\end{array}\right.$

The analysis is much simpler if we assume that the angle $\gamma$ and the length $L$ of the optics are such that $R \gg L \sin \gamma$. In this case, we can ignore the term dependent on $z_{\|}$in the second equation in Eq. (13), which simplifies to

$z=z(\boldsymbol{r})=\left\{\begin{array}{l}z_{\|}=\chi\left(|\boldsymbol{r}|-R_{0}\right) \\ z_{\perp}=R \angle \boldsymbol{r}\end{array}\right.$

where $\chi=\cos (\theta+\gamma) / \sin \theta$. The scaling factor of the roughness height is again $\sin \theta$ and Eq. (4) reads

$H(\boldsymbol{f})=\int A_{0}(r) A_{0}^{*}(|\boldsymbol{r}+\lambda \boldsymbol{f}|)\left\langle\mathrm{e}^{\frac{4 \pi \mathrm{i} \sin \theta}{\lambda}[h(z(\boldsymbol{r}))-h(z(\boldsymbol{r}+\lambda \boldsymbol{f}))]}\right\rangle \mathrm{d} \boldsymbol{r}$.

By assuming that the angle $\gamma$ is small enough for the optical surface to be locally considered a cylinder, the average in Eq. (15) gives

$H(\boldsymbol{f})=\int A_{0}(r) A_{0}^{*}(|\boldsymbol{r}+\lambda \boldsymbol{f}|) \mathrm{e}^{-\frac{16 \pi^{2} \sin ^{2} \theta}{\lambda^{2}}\left[\sigma^{2}-C(\delta z(\boldsymbol{r}, \boldsymbol{f}))\right]} \mathrm{d} \boldsymbol{r}$,

where $\delta \boldsymbol{z}(\boldsymbol{r}, \boldsymbol{f})=\boldsymbol{z}(\boldsymbol{r}+\lambda \boldsymbol{f})-\boldsymbol{z}(\boldsymbol{r})$. The functional dependence of $\delta z(\boldsymbol{r}, \boldsymbol{f})$ on $\boldsymbol{r}$ and $\boldsymbol{f}$ is determined from Eq. (14):

$$
\begin{aligned}
\delta z_{\|}(\boldsymbol{r}, \boldsymbol{f}) & =z_{\|}(\boldsymbol{r}+\lambda \boldsymbol{f})-z_{\|}(\boldsymbol{r})=\chi|\boldsymbol{r}+\lambda \boldsymbol{f}|-\chi|\boldsymbol{r}|, \\
\delta z_{\perp}(\boldsymbol{r}, \boldsymbol{f}) & =z_{\perp}(\boldsymbol{r}+\lambda \boldsymbol{f})-z_{\perp}(\boldsymbol{r})=R \angle(\boldsymbol{r}+\lambda \boldsymbol{f})-R \angle \boldsymbol{r} \\
& =R \arccos \left[\frac{\boldsymbol{r} \cdot(\boldsymbol{r}+\lambda \boldsymbol{f})}{r|\boldsymbol{r}+\lambda \boldsymbol{f}|}\right] .
\end{aligned}
$$

Equation (18) is valid when the anomaly of $\boldsymbol{r}+\lambda \boldsymbol{f}$ is higher than the anomaly of $\boldsymbol{r}$. Otherwise, a minus sign appears in front of Eq. (18). However, since the covariance is an even function of its arguments, we can ignore this change of sign.

An important conclusion can be derived from Eqs. (16)-(18). Since the pupil function $A_{0}(r)$ has cylindrical symmetry, the dependence of the integrand on the anomaly $\varphi_{f}$ of the vector $f$ is expressed by the scalar product $\boldsymbol{r} \cdot \boldsymbol{f}$ or, equivalently, by the function $\cos \left(\varphi-\varphi_{f}\right)$. By making the change of integration variable $\varphi \rightarrow \varphi+\varphi_{f}$, we see that the optical transfer function depends only on the modulus $f$ of the vector $f$ and, consequently, has cylindrical symmetry. We note that no assumptions have been made about the isotropy of the roughness covariance $C(\delta \boldsymbol{z})$. Regardless of the angular dependence of $C(\delta z)$, the optical transfer function and angle spread function inherit the cylindrical symmetry of the optics geometry.

A further observation can be made about the power spectral density of surface roughness. Since the covariance function is a periodic function of $z_{\perp}$ of period $2 \pi R$, the power spectral density $S(v)$ is discrete in the conjugate variable $v_{\perp}$. Only multiple values of $v_{\perp}=1 / 2 \pi R$ are allowed. However, this has limited practical implications since at this spatial frequency the entire formalism in which roughness is considered to be a stationary stochastic process breaks down. The theory is only applicable at spatial frequencies much higher than $v_{\perp}=1 / 2 \pi R$.

According to Eq. (9), to calculate the width of the angle spread function, we must evaluate the value of the Laplacian of the optical transfer function with respect to $f$ at the origin. This calculation is simplified by noting that the gradient $\nabla C(\delta z)$ vanishes at the origin, as shown in Appendix A. By taking into account that $C(0)=\sigma^{2}$ and $H_{0}(0)=1$, it follows from Eq. (16) that

$$
\begin{aligned}
\left.\Delta H(f)\right|_{f=0} & =\left.\int A_{0}(r) \Delta A_{0}^{*}(|\boldsymbol{r}+\lambda \boldsymbol{f}|)\right|_{f=0} \mathrm{~d} \boldsymbol{r} \\
& +\left.\frac{16 \pi^{2}}{\lambda^{2}} \sin ^{2} \theta \int\left|A_{0}(r)\right|^{2} \Delta C(\boldsymbol{f})\right|_{f=0} \mathrm{~d} \boldsymbol{r} .
\end{aligned}
$$

The first integral in Eq. (19) is simply $\left.\Delta H_{0}(f)\right|_{f=0}$ and, by means of Eq. (9), is equal to $-4 \pi^{2} \delta_{0}^{2}$, where $\delta_{0}$ is the angular width of the diffracted beam when scattering is ignored. The calculation of $\left.\Delta C(f)\right|_{f=0}$ is reported in Appendix A, which gives

$$
\begin{aligned}
\left.\Delta C(f)\right|_{f=0}= & \lambda^{2} \chi^{2} \cos ^{2} \varphi \frac{\partial^{2} C(0)}{\partial \delta z_{\|}^{2}} \\
& +2 \lambda^{2} R \chi \frac{\sin \varphi \cos \varphi}{r} \frac{\partial^{2} C(0)}{\partial \delta z_{\|} \partial \delta z_{\perp}} \\
& +\lambda^{2} R^{2} \frac{\sin ^{2} \varphi}{r^{2}} \frac{\partial^{2} C(0)}{\partial \delta z_{\perp}^{2}}
\end{aligned}
$$

where $\partial^{2} C(0) / \partial \delta z_{\|}^{2}$ actually means $\partial^{2} C(\delta z) /\left.\partial \delta z_{\|}^{2}\right|_{\delta z=0}$, etc. Since the pupil function has cylindrical symmetry and depends only on the modulus $r$ of the vector $\boldsymbol{r}$, the second term in Eq. (20) vanishes after integration over $\varphi$ because it is proportional to $\sin \varphi \cos \varphi$. The other two terms give a factor $\pi$ upon integration over $\varphi$ and, consequently,

$\left.\int_{0}^{2 \pi} \Delta C(f)\right|_{f=0} \mathrm{~d} \varphi=\pi \lambda^{2} \chi^{2} \frac{\partial^{2} C(0)}{\partial \delta z_{\|}^{2}}+\frac{\pi \lambda^{2} R^{2}}{r^{2}} \frac{\partial^{2} C(0)}{\partial \delta z_{\perp}^{2}}$.

The second derivatives at the origin of $C(\delta z)$ can be related to the power spectral density of surface roughness. Since the Fourier transform of $\Delta C(\delta z)$ is equal to $-4 \pi^{2} v^{2} S(v)$, we obtain

$\frac{\partial^{2} C(0)}{\partial \delta z_{\|}^{2}}=-4 \pi^{2} \int S(v) v_{\|}^{2} \mathrm{~d} v=-\rho_{\|}^{2}$

$\frac{\partial^{2} C(0)}{\partial \delta z_{\perp}^{2}}=-4 \pi^{2} \int S(v) v_{\perp}^{2} \mathrm{~d} v=-\rho_{\perp}^{2}$,

where $\rho_{\|}^{2}$ and $\rho_{\perp}^{2}$ are the variances of roughness slope along the direction of the optical axis and along circles normal to the optical axis, respectively. Inserting Eqs. (22) and (23) back into Eq. (21) and then Eq. (21) into Eq. (19), we obtain

$$
\begin{aligned}
\left.\Delta H(f)\right|_{f=0} & =-4 \pi^{2} \delta_{0}^{2}-16 \pi^{3} \sin ^{2} \theta \\
& \times\left[\chi^{2} \rho_{\|}^{2} \int_{0}^{\infty}\left|A_{0}(r)\right|^{2} r \mathrm{~d} r+R^{2} \rho_{\perp}^{2} \int_{0}^{\infty} \frac{\left|A_{0}(r)\right|^{2}}{r} \mathrm{~d} r\right] .
\end{aligned}
$$


The first integral in Eq. (24), multiplied by $2 \pi$, reduces to $H_{0}(0)=1$. To calculate the second integral in Eq. (24), we assume that the pupil function is constant and non-zero only for $R_{0} \leq r \leq R_{0}+d_{0}$. Since $A_{0}(r)$ has squared modulus normalized to unit integral, it is $A_{0}(r)=1 / \sqrt{\pi d_{0}\left(2 R_{0}+d_{0}\right)}$ for $R_{0} \leq r \leq R_{0}+d_{0}$ and $A_{0}(r)=0$ elsewhere. Thus, we obtain

$$
\begin{aligned}
\int_{0}^{\infty} \frac{\left|A_{0}(r)\right|^{2}}{r} \mathrm{~d} r & =\frac{1}{\pi d_{0}\left(2 R_{0}+d_{0}\right)} \int_{R_{0}}^{R_{0}+d_{0}} \frac{\mathrm{d} r}{r} \\
& =\frac{\ln \left[\left(R_{0}+d_{0}\right) / R_{0}\right]}{\pi d_{0}\left(2 R_{0}+d_{0}\right)} .
\end{aligned}
$$

By combining Eqs. (9), (24), and (25), the width of the angle spread function becomes

$\delta^{2}=\delta_{0}^{2}+2 \rho_{\|}^{2} \cos ^{2}(\theta+\gamma)+4 R^{2} \rho_{\perp}^{2} \sin ^{2} \theta \frac{\ln \left[\left(R_{0}+d_{0}\right) / R_{0}\right]}{d_{0}\left(2 R_{0}+d_{0}\right)}$,

where we have taken into account that $\chi=\cos (\theta+\gamma) / \sin \theta$. The total angular width of the reflected and scattered beam is then given by the quadratic sum of the beam width $\delta_{0}$ when surface roughness is ignored and a specific contribution due to roughness. Since we have assumed that $R \gg L \sin \gamma$, we also obtain $R_{0} \gg d_{0}$. Consequently, we can approximate $2 R_{0}+d_{0} \cong 2 R_{0}$ and $\ln \left[\left(R_{0}+d_{0}\right) / R_{0}\right] \cong d_{0} / R_{0}$. Thus, by defining $g=R / R_{0}$, Eq. (26) simplifies to

$\delta^{2}=\delta_{0}^{2}+2 \rho_{\|}^{2} \cos ^{2}(\theta+\gamma)+2 g^{2} \rho_{\perp}^{2} \sin ^{2} \theta$,

to first order in $d_{0} / R_{0}$. Equation (27) is independent of $d_{0}$. When both $\gamma$ and $\theta$ are small, Eq. (27) simplifies further to

$\delta^{2}=\delta_{0}^{2}+2 \rho_{\|}^{2}+2 g^{2} \rho_{\perp}^{2} \sin ^{2} \theta$.

By comparison with Eq. (10), we note that the contribution of roughness to the width of the angle spread function is half that found for the reflection on an almost flat surface.

As an example, we determine the roughness contribution $\delta_{\mathrm{sc}}$ to the rms beam width $\delta$ in the case of a single reflection mirror with surface roughness described by the isotropic power spectral density $S(v)=S_{0} v^{-2}$ (Church 1988). If $\psi$ is the half field of view, the cut-off spatial frequencies in Eqs. (22) and (23) are $v_{\|}=\sin \psi / \lambda \chi$ along the direction of the optical axis and $v_{\perp}=$ $\sin \psi / \lambda$ along the curved orthogonal direction, respectively. Here we assume that $g \cong 1$ and $\chi \cong 1 / \sin \theta$. Equations (22) and (23) then imply that

$$
\begin{aligned}
\rho_{\|}^{2}+\frac{\rho_{\perp}^{2}}{\chi^{2}} & =4 \pi^{2} S_{0} \int \frac{v_{\|}^{2}+v_{\perp}^{2} / \chi^{2}}{v_{\|}^{2}+v_{\perp}^{2}} \mathrm{~d} v \\
& =\frac{4 \pi^{2} S_{0}}{\chi^{3}} \int_{0}^{2 \pi} \mathrm{d} \varphi_{v} \int_{0}^{\sin \psi / \lambda} \frac{v_{\|}^{2}+v_{\perp}^{2}}{v_{\|}^{2} / \chi^{2}+v_{\perp}^{2}} v \mathrm{~d} v \\
& =\frac{2 \pi^{2} S_{0} \sin ^{2} \psi}{\lambda^{2} \chi^{3}} \int_{0}^{2 \pi} \frac{\mathrm{d} \varphi_{v}}{\sin ^{2} \varphi_{v} / \chi^{2}+\cos ^{2} \varphi_{v}},
\end{aligned}
$$

where we applied the change of integration variable $v_{\|} \rightarrow v_{\|} / \chi$ in the second equality and then integrated in polar coordinates. The last integral in Eq. (29) equals $2 \pi \chi$. Hence, from Eq. (28) we obtain that

$\delta_{\mathrm{sc}}^{2}=2 \rho_{\|}^{2}+\frac{2}{\chi^{2}} \rho_{\perp}^{2}=\frac{8 \pi^{3} S_{0}}{\lambda^{2}} \sin ^{2} \psi \sin ^{2} \theta$.

For $\theta=1^{\circ}, \psi=0.1^{\circ}, S_{0}=10^{-2} \mathrm{~nm}^{2}$, and $\lambda=1.24 \mathrm{~nm}$, corresponding to photon energy of $1 \mathrm{keV}$, Eq. (30) implies that $\delta_{\mathrm{sc}} \cong$ $39 \mu \mathrm{rad} \cong 8^{\prime \prime}$.
The extension of Eq. (27) to multiple uncorrelated surfaces is straightforward since the integrand in Eq. (16) is simply multiplied by an exponential factor for each surface (Stearns et al. 1998). In the presence of $N$ surfaces, each of roughness covariance $C_{k}(\boldsymbol{r})$, variance $\sigma_{k}^{2}$, slope variances $\rho_{\|, k}^{2}$ and $\rho_{\perp, k}^{2}$, incidence angle $\theta_{k}$, tapering angle $\gamma_{k}$, and scaling factor $g_{k}$, the optical transfer function becomes

$$
H(f)=\int A_{0}(r) A_{0}^{*}(|\boldsymbol{r}+\lambda \boldsymbol{f}|) \mathrm{e}^{-\frac{16 \pi^{2}}{\lambda^{2}} \sum_{k=1}^{N} \sin ^{2} \theta_{k}\left[\sigma_{k}^{2}-C_{k}\left(\delta z_{k}(\boldsymbol{r}, f)\right)\right.} \mathrm{d} \boldsymbol{r},
$$

and the beam width is now

$\delta^{2}=\delta_{0}^{2}+2 \sum_{k=1}^{N} \rho_{\|, k}^{2} \cos ^{2}\left(\theta_{k}+\gamma_{k}\right)+g_{k}^{2} \rho_{\perp, k}^{2} \sin ^{2} \theta_{k}$.

\section{Approximation of the angle spread function}

Equation (16) defines the optical transfer function as a complicated integral of the pupil function and the roughness covariance. In this section, we first develop a simpler approximation of the optical transfer function that provides the same expression as Eq. (27) for the beam width $\delta$. In the small roughness approximation, we also derive the angle spread function and express it in terms of an effective power spectral density obtained from the roughness power spectral density. Finally, the dependence of the rms beam width on the radiation wavelength and the grazing-incidence angle is studied in more detail.

\subsection{Approximate optical transfer function}

To develop an approximation to the optical transfer function that provides the correct expression given by Eq. (27) for the beam width $\delta$, we first note that we can approximate $r=|\boldsymbol{r}| \cong R_{0}$, since $A_{0}(r)$ is non-zero only in a thin annulus between $R_{0}$ and $R_{0}+d_{0}$. In addition, the factor $A_{0}(r) A_{0}^{*}(|\boldsymbol{r}+\lambda \boldsymbol{f}|)$ is equal to $1 / \pi d_{0}\left(2 R_{0}+\right.$ $d_{0}$ ) for $f=0$ and decreases with increasing $f$, becoming very small for $\lambda f \geq d_{0}$. We thus assume that $A_{0}(r) A_{0}^{*}(|\boldsymbol{r}+\lambda \boldsymbol{f}|)$ is independent of $\boldsymbol{r}$ for $0 \leq \lambda f \leq d_{0}$ and is negligible for $\lambda f>d_{0}$. We then write

$A_{0}(r) A_{0}^{*}(|\boldsymbol{r}+\lambda \boldsymbol{f}|) \cong \frac{H_{0}(f)}{\pi d_{0}\left(2 R_{0}+d_{0}\right)}$

where $H_{0}(f)$ starts at 1 for $f=0$ and decreases to zero at $f=$ $d_{0} / \lambda$. Thus, Eq. (16) can be simplified to

$H(f)=\frac{H_{0}(f)}{2 \pi} \mathrm{e}^{-\frac{16 \pi^{2} \sigma^{2} \sin ^{2} \theta}{\lambda^{2}}} \int_{0}^{2 \pi} \mathrm{e}^{\left.\frac{16 \pi^{2} \sin ^{2} \theta}{\lambda^{2}} C(\delta z(r, f))\right|_{r=R_{0}}} \mathrm{~d} \varphi$

where we have performed the integration over $r$. Since $0 \leq \lambda f \leq$ $d_{0}$ and $d_{0} \ll R_{0}$, then $\lambda f \ll R_{0}$, and consequently we obtain, to first order in $\lambda f / R_{0}$ (see also Eqs. (A.4) and (A.5) in Appendix A),

$$
\begin{aligned}
\left.\delta z_{\|}(\boldsymbol{r}, \boldsymbol{f})\right|_{r=R_{0}} & =\left.\chi(|\boldsymbol{r}+\lambda \boldsymbol{f}|-|\boldsymbol{r}|)\right|_{r=R_{0}} \cong \lambda f \chi \cos \varphi, \\
\left.\delta z_{\perp}(\boldsymbol{r}, \boldsymbol{f})\right|_{r=R_{0}} & =\left.R \arccos \left[\frac{\boldsymbol{r} \cdot(\boldsymbol{r}+\lambda \boldsymbol{f})}{r|\boldsymbol{r}+\lambda \boldsymbol{f}|}\right]\right|_{r=R_{0}} \\
& \cong \frac{\lambda f R}{R_{0}} \sin \varphi=\lambda f g \sin \varphi .
\end{aligned}
$$

Equation (31) then becomes

$H(f)=\frac{H_{0}(f)}{2 \pi} \mathrm{e}^{-\frac{16 \pi^{2} \sigma^{2} \sin ^{2} \theta}{\lambda^{2}}} \int_{0}^{2 \pi} \mathrm{e}^{\frac{16 \pi^{2} \sin ^{2} \theta}{\lambda^{2}} C(\lambda f \chi \cos \varphi, \lambda f g \sin \varphi)} \mathrm{d} \varphi$. 
In the small roughness approximation, when $16 \pi^{2} \sigma^{2} \sin ^{2} \theta \ll$ $\lambda^{2}$, Eq. (32) further simplifies to

$$
\begin{aligned}
H(f) & =H_{0}(f)+\frac{16 \pi^{2} \sin ^{2} \theta}{\lambda^{2}} H_{0}(f) \\
& \times \frac{1}{2 \pi} \int_{0}^{2 \pi} C(\lambda f \chi \cos \varphi, \lambda f g \sin \varphi) \mathrm{d} \varphi .
\end{aligned}
$$

The optical transfer function is then written in a rather standard form (Stearns et al. 1998; Harvey 1995b) as the sum of a term describing the diffraction pattern when surface roughness is ignored and a term accounting for roughness scattering. It is easy to verify that the expression of the beam width calculated from Eqs. (32) or (33) is identical to Eq. (27).

\subsection{Angle spread function and effective power spectral density}

When the small roughness approximation is valid, the angle spread function can be expressed in terms of an effective power spectral density obtained from the roughness power spectral density. By taking the 2-dimensional inverse Fourier transform of Eq. (33), we obtain

$P(\alpha)=P_{0}(\alpha)+\frac{16 \pi^{2} \sin ^{2} \theta}{\lambda^{4}} P_{0}(\alpha) * S_{\text {eff }}\left(\frac{\alpha}{\lambda}\right)$,

where $*$ denotes convolution and where $S_{\text {eff }}(v)$ is the inverse Fourier transform

$$
\begin{aligned}
S_{\text {eff }}(v) & =\mathfrak{F}^{-1}\left[\frac{1}{2 \pi} \int_{0}^{2 \pi} C(\chi z \cos \varphi, g z \sin \varphi) \mathrm{d} \varphi\right] \\
& =\int_{0}^{\infty} J_{0}(2 \pi v z) z \mathrm{~d} z \int_{0}^{2 \pi} C(\chi z \cos \varphi, g z \sin \varphi) \mathrm{d} \varphi .
\end{aligned}
$$

In the above equation, $J_{0}(x)$ is the Bessel function of the first type of order zero (Watson 1966). In a sense, $S_{\text {eff }}(v)$ is an effective power spectral density that can be written in terms of the true power spectral density $S(v)$ of surface roughness. The covariance in Eq. (34) corresponding to $S(v)$ is

$$
\begin{aligned}
C(\chi z \cos \varphi, g z \sin \varphi) & =\int S(\mu) \mathrm{e}^{2 \pi \mathrm{i} \chi \mu_{\|} z \cos \varphi+2 \pi \mathrm{i} g z \mu_{\perp} \sin \varphi} \mathrm{d} \mu, \\
& =\frac{1}{\chi g} \int S\left(\frac{\mu_{\|}}{\chi}, \frac{\mu_{\perp}}{g}\right) \mathrm{e}^{2 \pi \mathrm{i} \mu \| z \cos \varphi+2 \pi \mathrm{i} z \mu_{\perp} \sin \varphi} \mathrm{d} \mu,
\end{aligned}
$$

so that

$$
\int_{0}^{2 \pi} C(\chi z \cos \varphi, g z \sin \varphi) \mathrm{d} \varphi=\frac{2 \pi}{\chi g} \int S\left(\frac{\mu_{\|}}{\chi}, \frac{\mu_{\perp}}{g}\right) J_{0}(2 \pi \mu z) \mathrm{d} \mu .
$$

By inserting Eq. (35) into Eq. (34), we obtain

$S_{\text {eff }}(v)=\frac{2 \pi}{\chi g} \int S\left(\frac{\mu_{\|}}{\chi}, \frac{\mu_{\perp}}{g}\right) \mathrm{d} \mu \int_{0}^{\infty} J_{0}(2 \pi v z) J_{0}(2 \pi \mu z) z \mathrm{~d} z$.

Finally, by taking into account the identity (Watson 1966)

$$
\int_{0}^{\infty} J_{0}(2 \pi v z) J_{0}(2 \pi \mu z) z \mathrm{~d} z=\frac{\delta(\mu-v)}{4 \pi^{2} v},
$$

and by using polar coordinates for $\mu$, Eq. (36) becomes

$S_{\text {eff }}(v)=\frac{1}{2 \pi \chi g} \int_{0}^{2 \pi} S\left(\frac{v}{\chi} \cos \varphi, \frac{v}{g} \sin \varphi\right) \mathrm{d} \varphi$.

Thus, the effective power spectral density $S_{\text {eff }}(v)$ is given by the line integral of the roughness power spectral density along the ellipse of half-axes $v / \chi$ in the direction of the optical axis and $v / g$ along the curved orthogonal direction.

\subsection{Dependence of the beam width on the radiation wavelength}

To investigate the dependence of the scattered beam width $\delta$ on the radiation wavelength, we first note that $\delta_{0}$ depends linearly on $\lambda$. Since $\left.\Delta H_{0}(f)\right|_{f=0}=\left.\lambda^{2} \Delta \hat{H}_{0}(r)\right|_{r=0}$, where $\hat{H}_{0}(r)$ is the normalized autocorrelation of the complex pupil function defined by Eq. (1), and since $\hat{H}_{0}(r)$ is independent of $\lambda$, Eq. (9) implies that $\delta_{0}$ is proportional to the wavelength. The dependence of $\rho_{\|}^{2}$ and $\rho_{\perp}^{2}$ on $\lambda$ is more difficult to analyze and is related to the extreme spatial frequencies of surface roughness contributing to scattering. In the small roughness approximation, the angle spread function depends linearly on the effective power spectral density $S_{\text {eff }}(v)$, evaluated at $v=\alpha / \lambda$. In this case, the effective power spectral density is understood to be cut-off at both high and low spatial frequencies. The absolute maximum occurs at $1 / \lambda$, when $\alpha=|\alpha|=1$. The corresponding cut-offs of the power spectral density $S(v)$ are at $v_{\|}=1 / \lambda \chi$ along the direction of the optical axis and $v_{\perp}=1 / \lambda \mathrm{g}$ along the curved orthogonal direction. The integration domain is an ellipse with short-axis $2 / \lambda \chi$ and long-axis $2 / \lambda g$.

As an example, we consider an isotropic power-law dependence of the roughness power spectral density on the spatial frequency, $S(v)=S_{0} v^{-m}$ (Church 1988). The integrals in Eqs. (22) and (23) then give, assuming $g \cong 1,1 / \chi \cong \sin \theta$, and $m<4$,

$$
\begin{aligned}
\rho_{\|}^{2}+\frac{\rho_{\perp}^{2}}{\chi^{2}} & =4 \pi^{2} S_{0} \int \frac{v_{\|}^{2}+v_{\perp}^{2} / \chi^{2}}{\left(v_{\|}^{2}+v_{\perp}^{2}\right)^{m / 2}} \mathrm{~d} v \\
& =\frac{4 \pi^{2} S_{0}}{\chi^{3}} \int_{0}^{2 \pi} \mathrm{d} \varphi_{v} \int_{0}^{1 / \lambda} \frac{v_{\|}^{2}+v_{\perp}^{2}}{\left(v_{\|}^{2} / \chi^{2}+v_{\perp}^{2}\right)^{m / 2}} v \mathrm{~d} v \\
& =\frac{4 \pi^{2} S_{0}}{(4-m) \lambda^{4-m} \chi^{3}} \int_{0}^{2 \pi} \frac{\mathrm{d} \varphi_{v}}{\left(\sin ^{2} \varphi_{v} / \chi^{2}+\cos ^{2} \varphi_{v}\right)^{m / 2}}
\end{aligned}
$$

where we have used polar coordinates after the substitution $v_{\|} \rightarrow$ $v_{\|} / \chi$. By making the change of integration variable $y=\tan \varphi_{v} / \chi$, the last integral in Eq. (37) becomes

$$
\begin{aligned}
& \int_{0}^{2 \pi} \frac{\mathrm{d} \varphi_{v}}{\left(\sin ^{2} \varphi_{v} / \chi^{2}+\cos ^{2} \varphi_{v}\right)^{m / 2}} \\
= & 4 \chi^{m-1} \int_{0}^{\infty} \frac{\left(1 / \chi^{2}+y^{2}\right)^{m / 2-1}}{\left(1+y^{2}\right)^{m / 2}} \mathrm{~d} y=4 \chi^{m-1} I_{m}(\chi),
\end{aligned}
$$

where $I_{m}(\chi)$ is the last integral in Eq. (38) and is a slow varying function of $\chi$. For $m=1$ and $1 / \chi \ll 1$, the integral $I_{1}(\chi)$ is approximately equal to $\ln (2 \chi)$. For $2 \leq m<4$, we can ignore the term $1 / \chi^{2}$ in Eq. (38) and then $I_{m}(\infty) \cong \pi / m$. This value is exact for $m=2$ and $m=4$. Inserting Eqs. (37) and (38) back into Eq. (28) gives

$\delta^{2}=\delta_{0}^{2}+\frac{32 \pi^{2} S_{0} I_{m}(\chi)}{\lambda^{4-m}(4-m)} \sin ^{4-m} \theta$.

The scattering contribution is suppressed by a factor $\sin ^{2-m / 2} \theta$ and is proportional to $\lambda^{(m-4) / 2}$. Since we can assume that normally $1<m<3$, this contribution is a decreasing function of the wavelength. Taking into account that $\delta_{0}$ is proportional to the wavelength, Eq. (39) can be written as

$\frac{\delta}{\delta_{0}}=\sqrt{1+\sin ^{4-m} \theta\left(\frac{\lambda_{0}}{\lambda}\right)^{6-m}}$ 


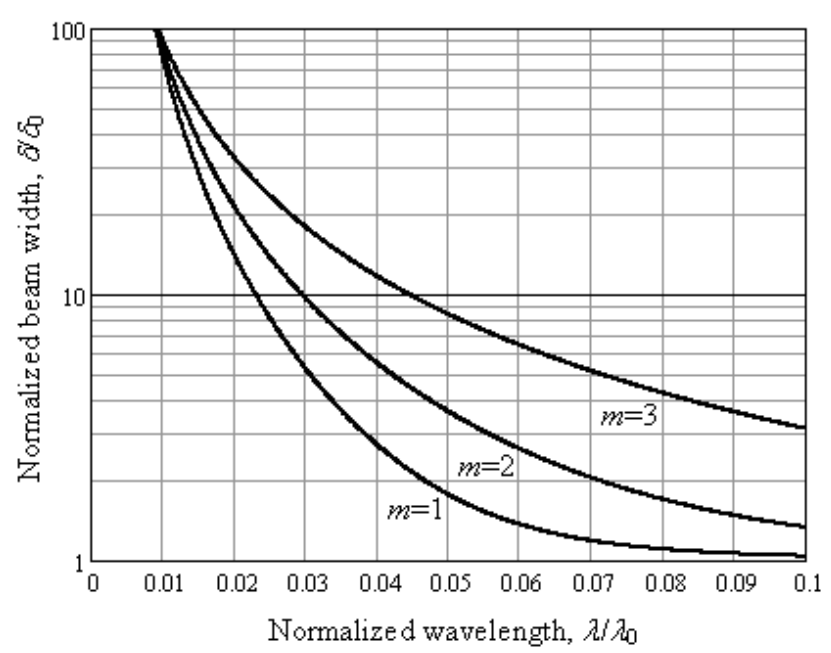

Fig. 2. Normalized beam width as a function of the normalized wavelength for a single reflection optics with $\psi_{0}=1^{\circ}$ and a power law expression of the roughness power spectral density. $m$ is the exponent of the power law.

where $\lambda_{0}=\left[32 \pi^{2} \lambda^{2} S_{0} I_{m}(\chi) /(4-m) \delta_{0}^{2}\right]^{1 /(6-m)}$ is a constant independent of the wavelength that depends only on the roughness power spectral density and the ratio of the beam width $\delta_{0}$ when roughness is ignored to the radiation wavelength $\lambda$. By means of Eq. (40), it is possible to study the dependence of the beam width on the radiation wavelength and the grazing-incidence angle. The use of the normalized quantities $\delta / \delta_{0}$ and $\lambda / \lambda_{0}$ simplifies the study and the graphical representation of the scaling property of $\delta$ with respect to $\lambda$.

Equation (40) is now studied in more detail for a grazingincidence mirror with one and two reflections, respectively. For a mirror with a single reflection and an object at infinity, $\theta \cong \psi_{0} / 2$, where $\psi_{0}$ is the angle of the principal ray at the focal plane with respect to the optical axis. Assuming that $\psi_{0}$ is small, Eq. (40) gives

$\frac{\delta}{\delta_{0}} \cong \sqrt{1+\frac{\sin ^{4-m} \psi_{0}}{2^{4-m}}\left(\frac{\lambda_{0}}{\lambda}\right)^{6-m}}$.

The ratio $\delta / \delta_{0}$ is plotted in Fig. 2 as a function of $\lambda / \lambda_{0}$ for a few values of $m$ when $\psi_{0}=1^{\circ}$. In a type I Wolter mirror for $\mathrm{X}$-ray astronomy, instead, there are two reflections, for which $\theta_{1} \cong \theta_{2} \cong \psi_{0} / 4$. In this case, Eq. (40) is extended to

$$
\frac{\delta}{\delta_{0}}=\sqrt{1+2 \sin ^{4-m} \theta_{1}\left(\frac{\lambda_{0}}{\lambda}\right)^{6-m}} \cong \sqrt{1+\frac{\sin ^{4-m} \psi_{0}}{2^{7-2 m}}\left(\frac{\lambda_{0}}{\lambda}\right)^{6-m}}
$$

where again $\psi_{0}$ is assumed to be small in the second equality. There is a reduction by a factor $2^{(3-m) / 2}$ in the scattering contribution to the beam width between Eqs. (41) and (42). The function given by Eq. (42), again for $\psi_{0}=1^{\circ}$, is shown in Fig. 3 .

\section{Conclusions}

In the framework of the scalar theory of Fourier diffraction, we have derived the width of the angle spread function of a grazingincidence optical system with cylindrical symmetry in the presence of roughness scattering. The derivation does not introduce further approximations in the theory except for assuming that

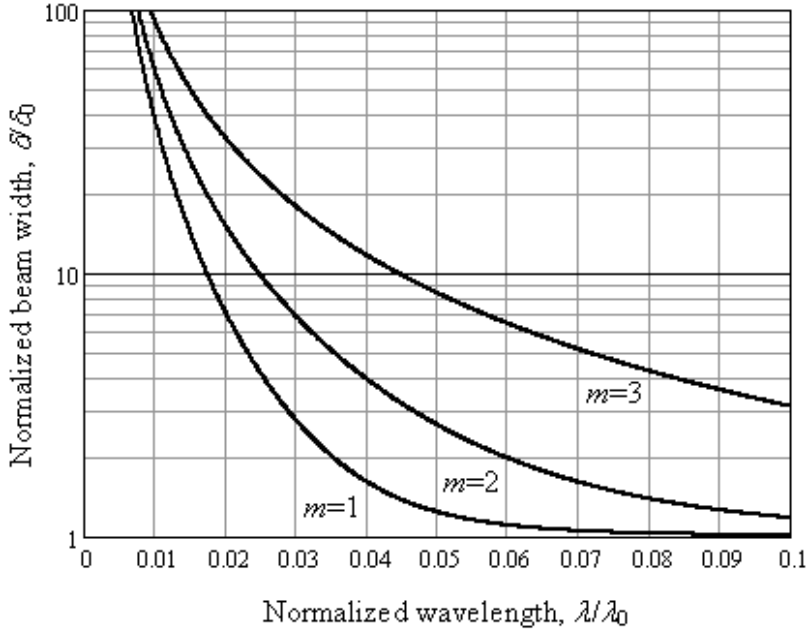

Fig. 3. Normalized beam width as a function of the normalized wavelength for a two reflections optics with $\psi_{0}=1^{\circ}$ and a power law expression of the roughness power spectral density. $m$ is the exponent of the power law.

the angle between the axial profile of the optics and the optical axis is small. Consequently, the results presented in this paper are more relevant to X-ray astronomy than other applications, such as EUV microlithography, that also use grazing-incidence mirrors with cylindrical symmetry but are characterized by quite large angles between the mirror profile and the optical axis.

When the small roughness approximation is applicable, the angle spread function itself can be expressed in a standard form as the convolution of the angle spread function of a perfectly smooth optics with an effective power spectral density related to the true power spectral density of surface roughness by line integration along an ellipse. This approximate expression for the angle spread function provides the correct expression for the width of the reflected and scattered beam and can be used for more accurate analyses of the scattering effects in X-ray telescopes. For example, it can be used to determine the encircled energy in the focal plane as a function of the encircling angular diameter.

\section{Appendix A: Calculation of $\left.\Delta C(f)\right|_{f=0}$}

We first show that the gradient of $C(f)$ vanishes at the origin. We work in polar coordinates for $\boldsymbol{f}$, a vector of modulus $f$ and anomaly $\varphi_{f}$. Since the optical transfer function, Eq. (16), has cylindrical symmetry and thus is independent of $\varphi_{f}$, we can simply ignore $\varphi_{f}$ and just set $\varphi_{f}=0$. The gradient of $C(f)$ is given by

$\nabla C(\boldsymbol{f}) \equiv \frac{\partial C(\boldsymbol{f})}{\partial f} \hat{\boldsymbol{f}}=\frac{\partial C(\delta \boldsymbol{z})}{\partial \delta z_{k}} \frac{\partial \delta z_{k}}{\partial f} \hat{\boldsymbol{f}}$,

where $\hat{\boldsymbol{f}}=|\boldsymbol{f}| / f$ and where summation over $k=\|, \perp$ is assumed. Equation (A.1) must be evaluated at $f=0$,

$\left.\nabla C(\boldsymbol{f})\right|_{f=0}=\left.\frac{\partial C(0)}{\partial \delta z_{k}} \frac{\partial \delta z_{k}}{\partial f} \hat{f}\right|_{f=0}$,

where $\partial C(0) / \partial \delta z_{k}$ means $\partial C(\delta z) /\left.\partial \delta z_{k}\right|_{\delta z=0}$. These partial derivatives, actually the components of the gradient of $C(\delta z)$, vanish at the origin. If $C(\delta z)$ is regular at the origin, this is simply because $C(\delta z)$ has its maximum at the origin. If, instead, $C(\delta z)$ has discontinuous derivatives at the origin, the quantity $\nabla C(0)$ must be 
understood to be the average of $\nabla C(\delta z)$ as $\delta z \rightarrow 0$. Since $C(\delta z)$ is an even function of $\delta z$, it follows again that $\nabla C(0)$ vanishes. To see that this is the correct interpretation, we note that the Fourier transform of $\nabla C(\delta z)$ is $2 \pi \mathrm{i} v S(\boldsymbol{v})$, where $S(\boldsymbol{v})$ is the power spectral density of surface roughness. Hence, the value at the origin of $\nabla C(\delta z)$ is

$\nabla C(0)=2 \pi \mathrm{i} \int S(v) v \mathrm{~d} v$.

Since $S(v)$ is an even function of $v$, the integral in the last equation vanishes as expected.

We next calculate the Laplacian of $C(f)$ at the origin, again ignoring $\varphi_{f}$. Using Eq. (A.1), we find that

$$
\begin{aligned}
\Delta C(\boldsymbol{f}) & \equiv \frac{1}{f} \frac{\partial C(\boldsymbol{f})}{\partial f}+\frac{\partial^{2} C(\boldsymbol{f})}{\partial f^{2}} \\
& =\frac{1}{f} \frac{\partial C(\delta \boldsymbol{z})}{\partial \delta z_{k}} \frac{\partial \delta z_{k}}{\partial f}+\frac{\partial C(\delta \boldsymbol{z})}{\partial \delta z_{k}} \frac{\partial^{2} \delta z_{k}}{\partial f^{2}}+\frac{\partial^{2} C(\delta \boldsymbol{z})}{\partial \delta z_{k} \partial \delta z_{j}} \frac{\partial \delta z_{k}}{\partial f} \frac{\partial \delta z_{j}}{\partial f} .
\end{aligned}
$$

When we evaluate the above expression at the origin, the first two terms in the second line vanish because of the above argument about the value of the gradient of $C(\delta z)$ at the origin, and consequently

$$
\left.\Delta C(\boldsymbol{f})\right|_{f=0}=\left.\frac{\partial^{2} C(0)}{\partial \delta z_{k} \partial \delta z_{j}} \frac{\partial \delta z_{k}}{\partial f} \frac{\partial \delta z_{j}}{\partial f}\right|_{f=0} .
$$

To calculate the right-hand side of Eq. (A.3), we use Eqs. (17) and (18) and we obtain

$$
\begin{aligned}
& \frac{\partial \delta z_{\|}}{\partial f}=\chi \frac{\partial}{\partial f}|\boldsymbol{r}+\lambda \boldsymbol{f}|=\lambda \chi \frac{r \cos \varphi+\lambda f}{|\boldsymbol{r}+\lambda \boldsymbol{f}|}, \\
& \frac{\partial \delta z_{\perp}}{\partial f}=R \frac{\partial}{\partial f} \arccos \left[\frac{\boldsymbol{r} \cdot(\boldsymbol{r}+\lambda \boldsymbol{f})}{r|\boldsymbol{r}+\lambda \boldsymbol{f}|}\right]=\lambda R r \frac{\sin \varphi}{|\boldsymbol{r}+\lambda \boldsymbol{f}|^{2}} .
\end{aligned}
$$

Evaluating these expressions at $f=0$ gives

$$
\begin{aligned}
& \left.\frac{\partial \delta z_{\|}}{\partial f}\right|_{f=0}=\lambda \chi \cos \varphi \\
& \left.\frac{\partial \delta z_{\perp}}{\partial f}\right|_{f=0}=\frac{\lambda R}{r} \sin \varphi .
\end{aligned}
$$

When Eqs. (A.4) and (A.5) are inserted back into Eq. (A.3), we obtain

$$
\begin{aligned}
\left.\Delta C(\boldsymbol{f})\right|_{f=0} & =\lambda^{2} \chi^{2} \cos ^{2} \varphi \frac{\partial^{2} C(0)}{\partial \delta z_{\|}^{2}} \\
& +2 \lambda^{2} R \chi \frac{\sin \varphi \cos \varphi}{r} \frac{\partial^{2} C(0)}{\partial \delta z_{\|} \partial \delta z_{\perp}}+\lambda^{2} R^{2} \frac{\sin ^{2} \varphi}{r^{2}} \frac{\partial^{2} C(0)}{\partial \delta z_{\perp}^{2}},
\end{aligned}
$$

which is Eq. (20) in the text.

\section{Appendix B: Validity of the shadowing effects approximation}

The theory that leads to Eq. (6) and on which the present paper is based, requires, to remain valid, some assumptions about the properties of the optical system under consideration and the statistical properties of surface roughness. These assumptions, which are listed in Sect. 2, include the requirement that multiple reflections and shadowing effects can be ignored. In this

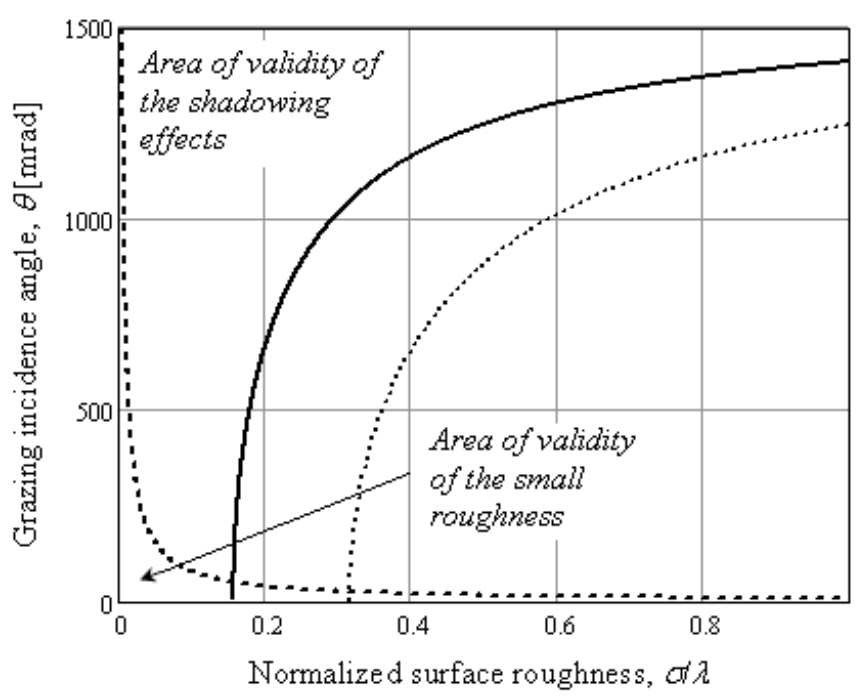

Fig. B.1. Limit of validity of the multiple scattering and shadowing effects approximation (solid trace) and of the small roughness approximation (dashed trace). A relaxed limit of validity of the approximation is also plotted (dotted trace).

Appendix, some considerations are discussed about the range of validity of this assumption and its relation to the small roughness approximation.

We can assume that multiple reflections and shadowing effects can be neglected if the grazing-incidence angle $\theta$ is larger than the rms slope error $\rho_{\|}$in the direction of the optical axis, or

$\theta>\arctan \rho_{\|}$.

Since $\rho_{\|}$is given by Eq. (22), it follows that

$\rho_{\|}^{2}=4 \pi^{2} \int v_{\|}^{2} S(v) \mathrm{d} \boldsymbol{v}<4 \pi^{2} v_{\|, \max }^{2} \int S(\boldsymbol{v}) \mathrm{d} \boldsymbol{v} \cong 4 \pi^{2} \frac{\sigma^{2}}{\lambda^{2}} \sin ^{2} \theta$,

where $v_{\|, \max }=\sin \theta / \lambda$. Hence, Eq. (B.1) becomes $\tan \theta>$ $2 \pi \sigma \sin \theta / \lambda$, or

$\cos \theta<\frac{\lambda}{2 \pi \sigma}$.

The limiting curve $\theta=\arccos (\lambda / 2 \pi \sigma)$ is plotted in Fig. B. 1 as a function of the normalized surface roughness $\sigma / \lambda$ (solid trace). Inequality (B.3) is valid above the curve. In the figure, the limiting curve for the validity of the small roughness approximation is also plotted (dashed trace). This is derived from the condition $4 \pi \sigma \sin \theta / \lambda \ll 1$ by assuming the equation $4 \pi \sigma \sin \theta / \lambda=0.1$ to be the limiting boundary. The small roughness approximation is valid below the dashed curve. The intersection of the two curves occurs at $\sigma / \lambda \cong 0.16$ and $\theta \cong 50 \mathrm{mrad}$.

It must be remarked that the above discussion is not limited to the analysis of the validity of the results presented in this paper but is applicable to the entire theory of the Fourier optics treatment of scattering from rough surfaces. Below $\sigma / \lambda=1 / 2 \pi$, the theory is always applicable, irrespective of the value of the incident angle. Above $\sigma / \lambda=1 / 2 \pi$, instead, the grazing-incidence angle above which the theory is valid increases rapidly with $\sigma / \lambda$. In particular, for grazing-incidence optics, the theory is not applicable even if the small roughness approximation holds. However, condition (B.3) is quite pessimistic because of the inequality sign in Eq. (B.2). Condition (B.3) also follows from Eq. (B.1) in the case of a single sinusoidal wave with rms value $\sigma$ 
and spatial frequency $v_{\|, \max }=\sin \theta / \lambda$. If a less restricting value of $v_{\|, \max }$ is used in Eq. (B.2), the limiting curve for the application of the theory shifts to higher values of the ratio $\sigma / \lambda$. As an example, the curve obtained by substituting $v_{\|, \max }$ with $v_{\|, \max } / 2$ into Eq. (B.2) is also plotted in Fig. B.1 (dotted trace).

\section{References}

Abbe, E. 1873, Archiv für Mikroskopische Anatomie, 9, 413

Aschenbach, B. 1988, Appl. Opt., 27, 1404

Bakshi, V., ed. 2008, EUV Lithography (Bellingham, WA: SPIE)

Barbee, T. 1986, Opt. Eng., 25, 898

Bookbinder, J., Smith, R., Hornschemeier, A., et al. 2008, in Proc. SPIE, 7011, 701102

Born, M., \& Wolf, E. 1980, Principles of Optics (Oxford, UK: Pergamon Press)

Braat, J. 1997, in Proc. SPIE, 3190, 59

Chase, R., \& Speybroeck, L. V. 1973, Appl. Opt., 12, 1042

Christensen, F., Hornstrup, A., \& Schnopper, H. 1988, Appl. Opt., 27, 1548

Church, E. 1988, Appl. Opt., 27, 1518

Church, E., \& Takacs, P. 1993, Appl. Opt., 32, 3344

Church, E., \& Takacs, P. 1995, Opt. Eng., 34, 353

Citterio, O., Bonelli, G., Conti, G., et al. 1988, Appl. Opt., 27, 1470

Foltyn, T., Bergmann, K., Braun, S., et al. 2004, in Proc. SPIE, 5533, 37

Friedrich, P., Bräuninger, H., Budau, B., et al. 2008, in Proc. SPIE, 7011, 70112T

Gaskill, J. 1978, Linear Systems, Fourier Transforms, and Optics (New York, NY: Wiley)

Gehrels, N., Chincarini, G., Giommi, P., et al. 2004, ApJ, 611, 1005

Giacconi, R., Reidy, W., Vaiana, G., VanSpeybroeck, L., \& Zehnpfennig, T. 1969, Space Sci. Rev., 9, 3
Gondoin, P., de Chambure, D., Katwijk, K. V., et al. 1994, in Proc. SPIE, 2279, 86

Goodman, J. 1968, Introduction to Fourier Optics (New York, NY: McGraw Hill) Harvey, J. 1995a, Appl. Opt., 34, 3715

Harvey, J. 1995b, in Proc. SPIE, 2515, 246

Harvey, J., Moran, E., \& Zmek, W. 1988, Appl. Opt., 27, 1527

Harvey, J., Lewotsky, K., \& Thompson, A. 1996, Opt. Eng., 35, 2423

Joensen, K., Voutov, P., Szentgyorgyi, A., et al. 1995, Appl. Opt., 34, 7935

Karabacak, T., Zhao, Y., Stowe, M., et al. 2000, Appl. Opt., 39, 4658

Papoulis, A. 1965, Probability, Random Variables, and Stochastic Processes (New York, NY: McGraw Hill)

Pareschi, G., \& Ferrando, P. 2005, Exp. Astr., 20, 139

Schellenberg, F., ed. 2008, Proc. SPIE: Emerging Lithographic Technologies XII, 6921

Schäfer, D., Nisius, T., Früke, R., et al. 2006, in Proc. SPIE, 6317, 631704

Silk, J. 1980, Annuals N.Y. Academy Sciences, 342, 116

Smith, F. 1963, Appl. Opt., 2, 335

Spiga, D. 2007, A\&A, 468, 775

Spiller, E. 1994, Soft X-ray Optics (Bellingham, WA: SPIE)

Spiller, E. 2003, in Proc. SPIE, 5193, 89

Stearns, D., Gaines, D., Sweeney, D., \& Gullikson, E. 1998, J. Appl. Phys., 84, 1003

Watson, G. 1966, A Treatise on the Theory of Bessel Functions, 2nd edn. (Cambridge, UK: Cambridge University Press)

Weisskopf, M., Tananbaum, H., Speybroeck, L. V., \& O'Dell, S. 2000, in Proc. SPIE, 4012, 2

Wolter, H. 1952a, Ann. Phys., 10, 286

Wolter, H. 1952b, Ann. Phys., 10, 94

Yamashita, K., Kuneida, H., Tawara, Y., et al. 1999, in Proc. SPIE, 3766, 327

Zhao, P., \& Spreybroeck, L. V. 2003, in Proc. SPIE, 4851, 124

Zocchi, F. 2009, Appl. Opt., 48, 436

Zocchi, F., \& Vernani, D. 2007, in Proc. SPIE, 6688, 66880B 